\title{
Rapid Response Preparedness for Management of Post-Covid Invasive Rhino-Orbito-Cerebral Fungal Infections: Experience from a Tertiary Care Government Institute in India
}

\author{
Amit Goyal $^{1}$ (D) Kapil Soni $^{1} \cdot$ Vidhu Sharma $^{1} \cdot$ Bikram Choudhury $^{1} \cdot$ Aman Verma $^{1}$ ' \\ Deepak Kumar $^{2}$ - Satyendra Khichar ${ }^{2}$ Ankita $\mathrm{Chugh}^{3} \cdot$ Binit Sureka $^{4}$. \\ Srikanth Srinivasan $^{5} \cdot$ Pankaj Bhardwaj $^{5} \cdot$ Manoj Kamal $^{6} \cdot$ Ashwini Agarwal $^{7}$. \\ Kavita Bhatnagar $^{8} \cdot$ Pradeep Kumar Bhatia $^{6} \cdot$ Mahendra Kumar Garg ${ }^{2} \cdot$ Sanjeev Misra ${ }^{9}$
}

Received: 20 June 2021 / Accepted: 4 July 2021 / Published online: 18 July 2021

(C) Association of Otolaryngologists of India 2021

\begin{abstract}
Sudden surge of Post Covid-19 Rhino-orbitomucormycosis cases has left entire ENT fraternity in the center of a war room. We present a quick administrative preparedness for this situation in a tertiary care Government Institute in India. This model may serve as a reference for other centers.
\end{abstract}

Keywords Mucormycosis · Preparedness · Pandemic · Covid-19 - Otorhinolaryngology residents

Amit Goyal

meetugoyal@yahoo.com; goyala@aiimsjodhpur.edu.in

1 Department of Otorhinolaryngology, All India Institute of Medical Sciences, Jodhpur, Rajasthan 342005, India

2 Department of Medicine, All India Institute of Medical Sciences, Jodhpur, India

3 Department of Dentistry, All India Institute of Medical Sciences, Jodhpur, India

4 Department of Diagnostic and Interventional Radiology, All India Institute of Medical Sciences, Jodhpur, India

5 Department of Community Medicine and Family Medicine, All India Institute of Medical Sciences, Jodhpur, India

6 Department of Anaesthesiology and Critical Care, All India Institute of Medical Sciences, Jodhpur, India

7 Department of Microbiology, All India Institute of Medical Sciences, Jodhpur, India

8 Department of Ophthalmology, All India Institute of Medical Sciences, Jodhpur, India

9 Department of Surgical Oncology, All India Institute of Medical Sciences, Jodhpur, India

\section{Introduction}

Rhinoorbitocerebral (ROC) Mucormycosis is the commonest form of invasive fungal infections. Though Union Health Ministry didn't release any official figures about mucormycosis in COVID-19 cases during the first wave of COVID-19, India's contribution to global cases of mucormycosis was approximately $71 \%$ in patients with COVID-19 based on published literature from December, 2019, to the start of April, 2021 [1]. Normally on an average 8-10 cases per annum of ROC Mucormycosis are being seen at a higher level center in India in pre-covid era, but there has been increased incidence during the second wave as compared with the first wave, with at least 14,872 cases as of May 28, 2021. This has led the Government of India to declare it as a notifiable disease under pandemic act and start strict surveillance [2].

The Indian Council of Medical Research released guidelines for the screening, diagnosis, and management of mucormycosis in patients with COVID-19. The most common causes attributed to the rise of mucormycosis in COVID-19 patients are uncontrolled or new onset diabetes, the excessive use of corticosteroids for immunosuppression, and long-term stay in the intensive care unit [3].

The management of ROC Mucormycosis is essentially a multidisciplinary effort which requires an Otorhinolaryngologist, Ophthalmologist, Neurologist/Neurosurgeon, Anesthetists, Critical care specialists with intensive care unit (ICU) facility, Plastic surgeons, Rehabilitation professionals, Internal Medicine specialists and Endocrinologists complimented by well-equipped investigative Departments like radiology, microbiology, laboratory medicine and pathology.

Ear nose and throat (ENT) specialty is in the center of management of these cases, which requires prompt surgery 
(multiple surgeries, if needed) and regular good postoperative care with anti-fungal medications to be continued for months for successful outcome and good glycemic control. It is followed by vigorous rehabilitation on case to case basis. Despite adequate treatment, morbidity and mortality rates remain high.

We also faced similar situation where we had to mobilize all our available resources for optimal care of these patients. Conventionally ENT Departments do not have huge manpower in terms of faculty and residents to cater case load of 8-10 new cases every day for many weeks, so adequate planning for optimal patient care and outcome with adequate rest to each team member was the key. Since day one it was looked at as a long war rather than a short term battle.

Due to second wave of Covid, we had our routine elective operation theatres (OTs) already suspended and we were catering out-patients departments (OPD) via telemedicine.

The following system was put in place immediately to fight with this mucormycosis surge at our Institute.

\section{Manpower Mobilization}

The residents of ENT Department were divided into teams for OPD, emergency, in-patient department (IPD), Covid19 postitive ward with mucormycosis patients and OT. OT team was given maximum manpower and they were further divided on the basis of Covid-19 and non-Covid OT as well as shifts were defined for them. One faculty was given the charge of OT scheduling for optimal utilization of OT slots and all the faculty joined to operate.

OPD, Emergency, IPD and Covid-mucor ward residents were also supervised by faculty In-charge of these areas. OPD team was given the responsibility of daily post-operative care of these patients for cavity care and limited debridement on ENT Workstation, if required.

Set protocol was defined for all places to reduce miscommunication and mismanagement. Emergency team would examine any suspected patient clinically and take a punch biopsy in emergency area itself. Urgent CT PNS would be done and then only patient used to be shifted to ward according to COVID status.

Ward team was responsible for all ward management as well as pre-operative work-up of the patient. This was communicated to OT team on daily basis and OT list was finalized accordingly.

OT team was able to run 2-3 OTs for $24 \mathrm{~h}$ a day, posting cases for primary or revision surgery. All attempts were made to keep the pendency for surgery to less than 1 day.

\section{Space allocation for IPD}

Immediately dedicated ward for Mucormycosis cases was allotted in Covid and Non-Covid areas and residents from ward team were posted. A total of 100 beds were defined for Mucor patients (including Covid and Non-covid areas).

\section{Emergency protocol}

History, clinical examination and Diagnostic nasal endoscopy were performed immediately on presentation. Endoscopic nasal turbinate punch biopsy was taken from suspicious area and sent for KOH. RT-PCR for Covid 19 was sent and urgent radiology (Contrast CT PNS) was done. Only then the patient was shifted to IPD area with full work-up done, ready for earliest debridement.

\section{OT Management}

The main focus was to get optimal output in terms of number of cases and minimizing complications. Two OTs were running round the clock for initial two weeks. Faculty used to operate and supervise other OTs. The residents were called in shifts to reduce exhaustion and fatigue. All the efforts were made to minimize OT pendency and surgical waiting time.

Two anaesthesia teams were deputed round the clock dedicated to mucormycosis cases.

This way, within 5 days, we were able to bring our surgical waiting period to less than $24 \mathrm{~h}$.

\section{Ward management}

Ward teams were responsible for regular care. OPD team was additionally responsible for postoperative cavity care on ENT Workstation. Separate resident's team was looking after Covid-Mucor wards under supervision of one faculty. Ophthalmic and Medicine teams used to take daily rounds twice a day. Combined rounds were taken in morning hours. This closely monitored the ophthalmic status and suitable candidates were offered administration of Transcutaneous Retrobulbar Amphotericin B also. Medicine faculty and residents were taking care of all the co-morbidities of the patients including strict blood sugar control, electrolyte disturbances, and renal functions monitoring etc. which is very common due to diabetes and anti-fungal therapy. Many patients were Covid-19 positive requiring lung status monitoring and management. 
Initially, the antifungal therapies were not available in sufficient quantity, so judicious use of antifungal therapy was enforced by a group of three clinicians, and it was based purely on the clinical condition and requirements of the patients. Efforts were made that every patient receive adequate antifungal therpay regularly. We categorized the patients into Low risk (limited disease, no intraorbital/intracranial involvement and good glycemic control) versus High risk (extensive involvement, intraorbital/intracranial involvement, poor glycemic control). Low risk patients were put on oral Posaconazole and high risk were given intravenous liposomal amphotericin B. Strict clinical and radiological monitoring of the disease was done.

The whole focus was to maintain maximum patients in best suitable way till the drug supply improves.

\section{Administrative systems}

Quickest possible administrative approvals were accorded for urgent purchase of additional instruments sets and other requirements. War level efforts were made to procure antifungal therapy with coordination from state and central agencies, and from whatever sources possible. Manpower mobilization for nursing and other staff was also done immediately to provide adequate support.

A separate reporting team was made to report all the cases on Government portal since it is declared a notifiable disease. It reduced the burden on clinical teams.

The residents in all teams were rotated on weekly basis to break the monotony and exhaustion from many days working condition, and due to huge workload, the members of one team were not able to meet other team members for many days.

This is an unprecedented situation to see a flood of mucormycosis patients and many of them are quite young. With excellent support from the administration and other Departments, we were able to set up an adequate and protocolized facility to take adequate care of these patients. Everything was setup within two days' time. We are able to sustain the workflow with optimal care and minimizing exhaustion to already limited workforce.

This model may provide a quick reference for administration of Mucormycosis management facility in different set ups, based on their needs and strengths.

\section{References}

1. Raut A, Huy NT (2021) Rising incidence of mucormycosis in patients with COVID-19: another challenge for India amidst the second wave? (Published online). https://doi.org/10.1016/S2 213-2600(21)00265-4

2. John TM, Jacob CN, Kontoyiannis DP (2021) When uncontrolled diabetes mellitus and severe COVID-19 converge: the perfect storm for mucormycosis. J Fungi 7:298

3. Indian Council of Medical Research (2021) Evidence based advisory in the time of COVID-19 (screening, diagnosis \& management of mucormycosis). https://www.icmr.gov.in/pdf/c ovid/techdoc/Mucormycosis_ADVISORY_FROM_ICMR_In_CO VID19_time.pdf. Accessed May 282021

Publisher's Note Springer Nature remains neutral with regard to jurisdictional claims in published maps and institutional affiliations. 